\title{
Event Termination Cue Generates a Field in which Perceived Time Dilates
}

Running head: Termination Cues Generate Time Dilation

Seonggyu Choe and Oh-Sang Kwon

Department of Biomedical Engineering, Ulsan National Institute of Science and Technology, Ulsan 44919, Republic of Korea

\section{Corresponding author}

Oh-Sang Kwon

50 UNIST-gil, Ulsan 44919, Republic of Korea

Telephone: +82-52-217-2735

Fax: +82-52-217-2708

oskwon@unist.ac.kr

$\underline{\text { Keywords }}$

Time dilation, Time reproduction task, Attention field, Subjective time 


\begin{abstract}
The perceived duration of time does not veridically reflect the physical duration but is distorted by various factors, such as the stimulus magnitude or the observer's emotional state. Here, we showed that knowledge about an event's termination time is another significant factor. We often experience time passage differently when we know that an event will terminate soon. To quantify this, we asked 33 university students to report a rotating clock hand's duration with or without a termination cue that indicated the position at which the clock hand disappeared. The results showed that the presence of the termination cue dilated perceived durations, and the dilating effect was larger when the stimulus duration was longer, or the speed of the rotating stimulus was slower. A control experiment with a start-cue excluded the possibility that the cue's mere existence caused the results. Further computational analyses based on the attention theory of time perception revealed that the size of dilation is best explained by neither an event's duration nor the distance traveled by the clock hand, but by how long the clock hand spends time near the termination cue. The results imply that an event termination cue generates a field in which the perceived time dilates.
\end{abstract}




\section{Introduction}

We sometimes know when an event will end, but sometimes we do not. For instance, students know when a class will end, but usually do not know when a principal's speech will end. We know when a cooking timer will sound but do not know when water in a pot will boil. When we have knowledge about the termination time of an event, we experience the passage of time differently, especially when the time approaches the end (e.g., final moments of a 5-km run). Despite the ecological relevance, studies regarding the effect of event termination information on time perception are lacking. We examined how event termination cues affect perceived duration of a time interval using a timer-like stimulus.

Numerous studies have demonstrated that diverse factors can alter the perceived duration of a time interval (Allan, 1979; Buhusi \& Meck, 2009). A moving stimulus is perceived to last longer than a stationary stimulus (Brown, 1995; Lhamon \& Goldstone, 1975), familiar words are judged as lasting longer than unfamiliar ones (Witherspoon \& Allan, 1985), and auditory stimuli produce a longer perceived duration than visual stimuli (Goldstone \& Lhamon, 1974). The physical magnitude of a target stimulus affects perceived duration (Dormal et al., 2006; Oliveri et al., 2008). The duration of a stimulus is perceived to be longer when the stimulus size is larger (Cai et al., 2018; Ono \& Kawahara, 2007; Xuan et al., 2007), the stimulus speed is faster (Kaneko \& Murakami, 2009; Li et al., 2015; Makin et al., 2012), the sound is louder, or the pitch is higher (Wearden et al., 2006). The eventfulness, the number of changes in the stimulus features, is positively related to perceived duration (Block \& Reed, 1978; Boltz, 1995; Faber \& Gennari, 2015). The occurrence of unexpected stimuli in a series of similar stimuli expands perceived duration (Birngruber et al., 2014; Cai et al., 2015; Pariyadath \& Eagleman 2007; Tse et al. 2004; Ulrich et al., 2006).

The attention theory of time perception provides a concise explanation for a large volume of the phenomena (Brown, 1997; Brown, 1985; Coull \& Nobre, 1998; Matthews \& 
Meck, 2016; Wittmann, 2013). The theory states that the stimulus that attracts more attention is perceived to last longer. According to the theory, stimuli with salient features (e.g., movement or familiarity) or larger magnitudes (e.g., faster, bigger, louder, or more eventful) draw more attention and, consequently, are perceived to last longer. Unexpected stimuli in the oddball task drew attention and lengthened the perceived duration. Endogenous devotion of attention to a secondary task reduced the amount of attention devoted to the main task and shortened perceived duration of a target stimulus (Bangert et al., 2020; Brown, 1985; Fortin et al., 1993; Franssen \& Vandierendonck, 2002).

Based on the attention theory of time perception, one may conjecture that a cue informing an event's termination time may attract the attention around the cue and, in turn, affects the perceived duration. To test the conjecture, we asked participants to report the duration of a rotating clock hand presented for 0.5 to 3 seconds. The key manipulation was the presence or absence of the termination-cue that indicated the location at which the rotating clock hand disappeared. When the cue was present, participants observed the rotating clock hand that approached the cue, knowing that the clock hand would disappear at the cue location. When the cue was absent, participants observed a rotating clock hand without knowing when the clock hand would disappear. The results showed that the event termination cue dilated the perceived duration, and the dilation size depended on the stimulus duration and speed. Based on the attention theory of time perception, we devised a computational model that assumes the event termination cue generates a spatial field in which the stimulus duration dilates. The performance of the model closely matched the pattern of the observed data. 


\section{Methods}

\section{Participants}

Thirty-three university students (Experiment 1: 15, Experiment 2: 18), who were naïve to the experimental purpose, participated in the experiments. They had normal or corrected-to-normal vision and provided informed consent. The study was approved by the Ulsan National Institute of Science and Technology Institutional Review Board. All participants signed a written consent form before the experiment and received a monetary reward regardless of their task performance. The sample size was chosen based on three previous studies that involved similar time reproduction tasks ( 8 participants, Chang \& Jazayeri, 2018; 14 participants, Cicchini et al., 2012; 6 participants, Jazayeri \& Shadlen, 2010). Mean responses for each participant were calculated individually for each experimental condition, where the responses that are larger than 2.5 standard deviations were excluded. One participant was excluded from the analysis.

\section{Apparatus}

The stimuli were created in MATLAB and the Psychophysics Toolbox (Brainard, 1997; Pelli, 1997), and were presented by a digital light processing projector (PROPixx; 1920 $\times 1080 ; 120 \mathrm{~Hz}$, linear gamma). The viewing distance was $1.6 \mathrm{~m}$, and head movements were restrained by a head and chin rest.

\section{Procedures}

Throughout the experiments, participants were instructed to fixate their eyes on a white fixation point $\left(0.5^{\circ}\right)$ at the center of the screen. After a blank display with an interval uniformly distributed from 1000 to $1500 \mathrm{~ms}$, a white bar $\left(4.5 \times 0.3^{\circ}\right)$ appeared and rotated for the to-be-timed duration, mimicking the motion of a clock hand. Stimulus durations were drawn from a discrete uniform distribution ranging from 500 to $3000 \mathrm{~ms}$ with 10 linearly 
spaced intervals, and there were 5 angular speeds $(24,48,72,96$, and $120 \%$ s). A combination of 10 durations and 5 speeds made 50 different distances. The participants underwent 8 trials for each of the 50 conditions, and the presentation order of the trials was randomized. After the rotating bar arrived at the final position and then disappeared, participants pressed the space bar for the previously presented duration and then released it to reproduce the duration of the stimulus. No feedback was provided on the accuracy of their responses.

There were two conditions in Experiment 1. In the termination-cue condition, all trials had a red marker $\left(0.5 \times 0.3^{\circ}\right)$ placed at the termination point (Fig. 1a). This marker appeared and disappeared simultaneously with the rotating stimulus. The marker was removed for the no-cue condition (Fig. 1b, right panel). Each condition involved 5 blocks of 80 trials with an intermission of at least 2 min between blocks and took approximately 1 hour. Participants performed one condition per day, and the order of the experimental conditions was randomly assigned. In Experiment 2, participants completed three conditions. Two conditions were replications of Experiment 1. In the third condition, the start-cue condition, the same red marker was given at the initial point of the rotating stimulus (Fig. 1b, middle panel). Except for the location of the marker, the procedure was the same as that in the termination-cue condition. The start-cue condition was designed to examine whether the mere existence of an additional visual object, which does not provide information on the termination time, affects the reproduced duration. Participants performed one condition per day, and the order of the experimental conditions was randomized across participants. Thus, it took three separate days for each participant to complete all the conditions. 


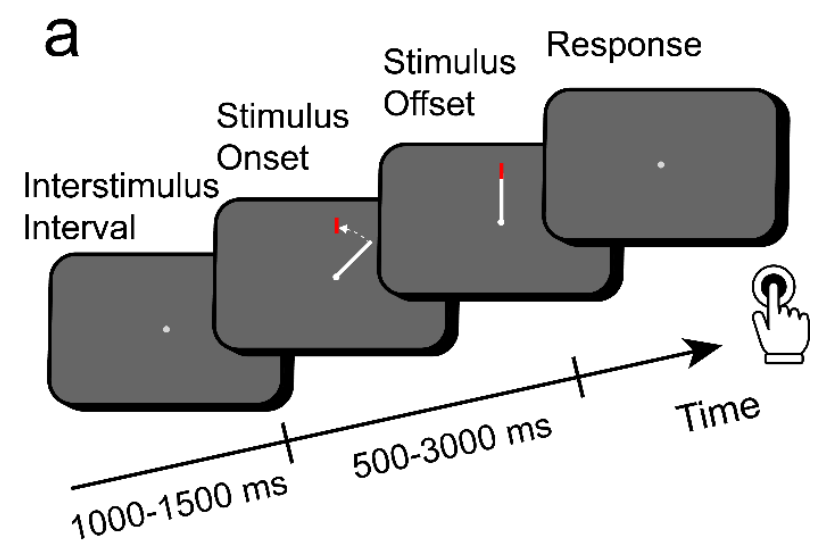

b

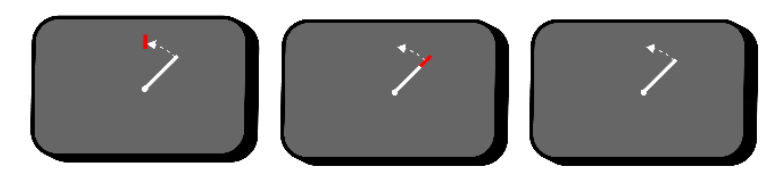

Termination-Cue Start-Cue No Cue

Fig. 1 A schematic illustration of a single trial. (a) The termination-cue condition in Experiment 1 . There is a red marker placed at the final position to indicate the termination point. (b) Three experimental conditions in Experiment 2. The relative position of the cue and the rotating clock hand at the beginning of each trial is shown (Left: termination-cue, middle: start-cue, right: no cue). Between the termination-cue condition and the start-cue condition, there is no difference in terms of the visual composition within a display.

\section{Models of cue effect}

We considered three models to formally describe the pattern of cue effects: time model, space model, and time in space model. In the time model, the stimulus duration determines the size of the cue effect, $C E(t)$, which is expressed as a function of the stimulus duration, $t: C E(t)=F_{\text {Time }}(t)$. The cue effect is the amount of subjective time distortion due to the termination- or start-cue. In the space model, the travel distance determines the size of the cue effect, $C E(d)$, which is expressed as a function of the travel distance, $d: C E(d)=$ $F_{\text {Space }}(d)$. In the time in space model, the cue effect, $C E(d, s)$, is affected by both the travel distance, $d$, and the speed, $s$, at which the clock hand travels. The size of the cue effect is expressed as follows:

$$
C E(d, s)=F_{\text {Tins }}(d) / s
$$


Intuitively, the time in space model assumes that the cue generates a spatial field where the perceived duration is distorted, and the size of distortion changes proportionally to the time spent in the field. The time spent in each unit distance is proportional to the reciprocal of the speed in the unit distance. As the speed is constant in our experiment, the cue effect can be obtained by dividing the $F_{\text {Tins }}(d)$ by speed, $s$, as in equation [1]. One may conceive a general form of the model that can deal with varying speeds (see the supplementary information).

Fig. 2 conceptually illustrates the cue effects predicted by the three models (a: time, b: space, and c: time in space). For illustrative purposes, we assumed an arbitrary function for three cue effect functions, $F_{\text {Time }}, F_{\text {Space }}$, and $F_{\text {Tins }}$. Note that the shape of the function determines the model's performances in all three models. We considered ten levels of stimulus durations and five levels of stimulus speeds, which generated 50 conditions. In the time model, the stimulus duration solely determined the cue effects, and the stimulus speed did not affect them at all. Five data points representing five different speeds for a given duration overlapped (Fig. 2a). In the space model, the stimulus distance solely determines the cue effect regardless of the travel time or speed (Fig. 2b). In the time in space model, stimulus distance determines the pattern of cue effects, but the stimulus speed also affects the cue effects in such a way that the cue effects increase inversely proportional to the speed. In principle, $F_{\text {Time }}, F_{\text {Space }}$, and $F_{\text {Tins }}$ can be any functions. However, we parameterized them using a cubic function to limit the search space in the model fitting (see supplementary information). We obtained the best-fitting parameters by applying the maximum-likelihood estimation and compared the performances of the three models by computing the Akaike information criterion (AIC). Details of the model fitting and model comparison procedures are described in detail in the model specification of the supplementary information. 


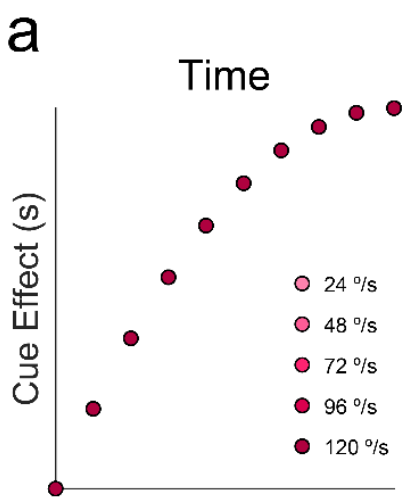

Duration (s) b

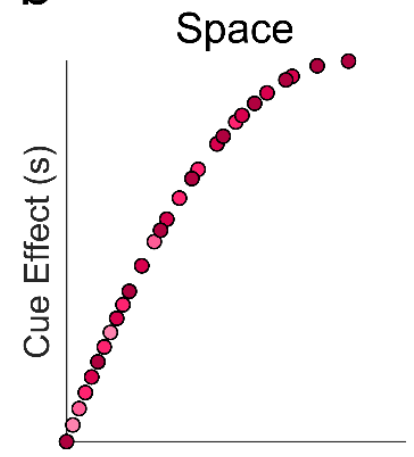

Distance $(\mathrm{deg})$

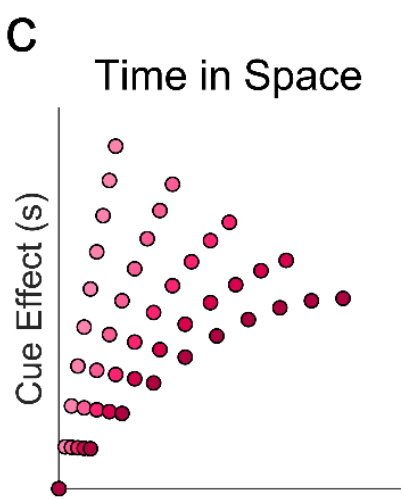

Distance (deg)

Fig. 2 Schematic illustration of the three models' performances for ten levels of stimulus durations and five levels of stimulus speeds. (a) The time model predicts that the stimulus duration will determine the size of cue effect and the stimulus speed will not affect the size. (b) The space model predicts that the travel distance will determine the size of cue effect. (c) The time in space model predicts that a slower stimulus will show a larger cue effect given the same distance.

\section{Results}

\section{Event termination cue dilates perceived duration}

We first examined whether the three factors of the experiments (cue type, speed, and stimulus duration) systematically affected the response error computed by subtracting the physical durations from the reproduced durations. The results of repeated measures ANOVA for Experiment 1 (Fig. 3a and 3b) show that the response error of the termination-cue condition was significantly larger than that of the no-cue condition $\left(\mathrm{F}_{1,14}=10.48, \eta_{p}^{2}=.63, \mathrm{p}\right.$ $=.006)$. On average, the termination-cue dilated the reproduced durations by $11.7 \%$. The main effect of the stimulus duration on the response error was statistically significant $\left(\mathrm{F}_{9,126}\right.$ $\left.=16.18, \eta_{p}^{2}=.79, \mathrm{p}<.001\right)$, and the linear regression analysis confirmed the decreasing tendency of response error was statistically significant (termination-cue: $t_{14}=-3.29$, Cohen's $d=.850, \mathrm{p}=.005$; no-cue: $\mathrm{t}_{14}=-4.60$, Cohen's $\left.d=1.188, \mathrm{p}<.001\right)$. The overestimation of relatively small durations and underestimation of relatively large durations are consistent with the results of various magnitude estimation tasks (Gibbon 1977; Hollingworth, 1910; 
Jazayeri \& Shadlen, 2010; Kwon \& Knill 2013; Petzschnner \& Glasauer, 2011). There was a statistically significant speed effect $\left(\mathrm{F}_{4,56}=29.70, \eta_{p}^{2}=.57, \mathrm{p}<.001\right)$. Consistent with the existing literature (Kaneko \& Murakami, 2009; Li et al., 2015; Makin et al., 2012), the response error increased as the stimulus speed increased in both conditions, and the trend was statistically significant (termination-cue: $\mathrm{t}_{14}=4.90$, Cohen's $d=1.266, \mathrm{p}<.001$; no-cue: $\mathrm{t}_{14}$ $=6.09$, Cohen's $d=1.573, \mathrm{p}<.001)$. There was a significant interaction between cue type and duration $\left(\mathrm{F}_{9,126}=5.12, \eta_{p}^{2}=.20, \mathrm{p}<.001\right)$ and the cue type and speed $\left(\mathrm{F}_{4,56}=12.14, \eta_{p}^{2}\right.$ $=.15, \mathrm{p}<.001)$. Detailed analyses and interpretations of these interactions are presented in the following section.

The results of Experiment 2 were largely analogous to those of Experiment 1 (Fig. 3c and $3 \mathrm{~d})$. There were statistically significant main effects of cue type $\left(\mathrm{F}_{2,34}=7.54, \eta_{p}^{2}=.65, \mathrm{p}\right.$ $=.002)$, duration $\left(\mathrm{F}_{9,153}=29.85, \eta_{p}^{2}=.88, \mathrm{p}<.001\right)$ and speed $\left(\mathrm{F}_{4,68}=41.19, \eta_{p}^{2}=.79, \mathrm{p}\right.$ $<.001)$. Tukey's test revealed that the difference between termination-cue and no-cue conditions was statistically significant $(\mathrm{p}=.001)$; however, the differences between start-cue and no-cue conditions $(\mathrm{p}=.157)$ or termination-cue and start-cue conditions $(\mathrm{p}=.130)$ were not statistically significant. Linear regression analyses showed that the response error increased as the stimulus duration decreased (termination-cue: $\mathrm{t}_{17}=-4.33$, Cohen's $d=1.020$ $\mathrm{p}<.001$; start-cue: $\mathrm{t}_{17}=-6.59$, Cohen's $d=1.553, \mathrm{p}<.001$; No-cue: $\mathrm{t}_{17}=-5.43$, Cohen's $d$ $=1.279, \mathrm{p}<.001$ ) or the speed increased (termination-cue: $\mathrm{t}_{17}=4.68$, Cohen's $d=1.103, \mathrm{p}$ $<.001$; Start-cue: $\mathrm{t}_{17}=6.79$, Cohen's $d=1.601, \mathrm{p}<.001$; No-cue: $\mathrm{t}_{17}=7.01$, Cohen's $d=$ $1.653, \mathrm{p}<.001)$. The cue type had a significant interaction with duration $\left(\mathrm{F}_{18,306}=3.64, \eta_{p}^{2}\right.$ $=.18, \mathrm{p}<.001)$ and speed $\left(\mathrm{F}_{8,136}=11.56, \eta_{p}^{2}=.16, \mathrm{p}<.001\right)$. 


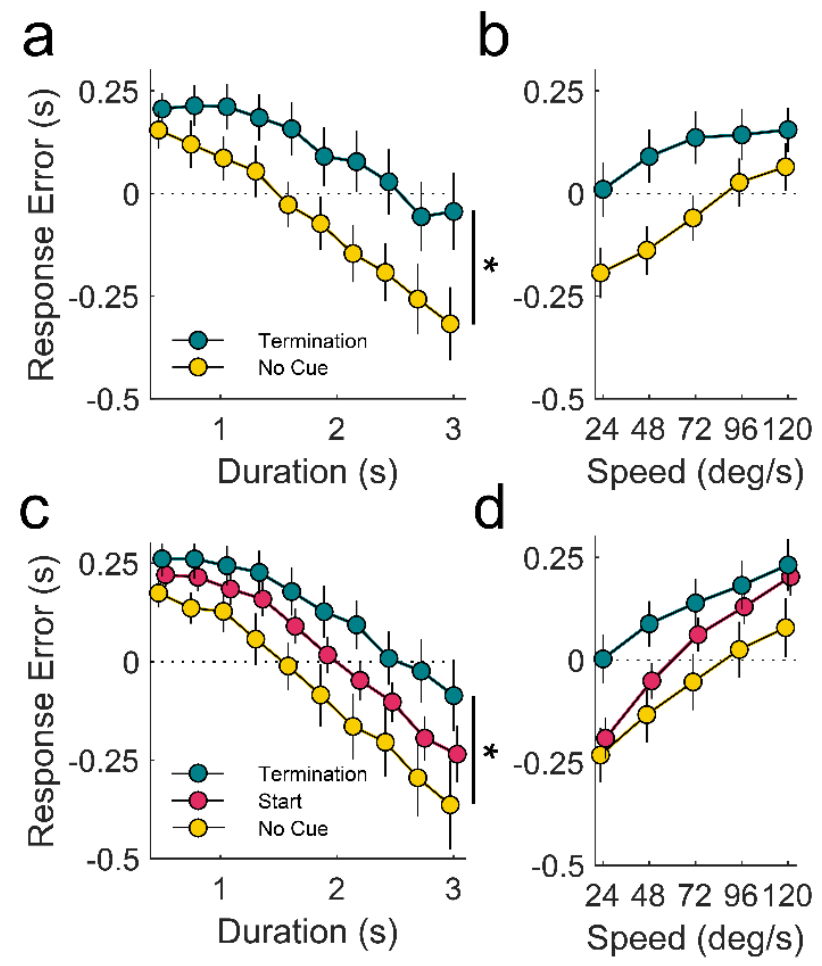

Fig. 3 The response errors as a function of the stimulus duration (a and $\mathbf{c}$ ) and the stimulus speed (b and d) observed in Experiment 1(a and b) and Experiment 2 (c and d). Green, red, and yellow indicate the termination-cue, the start-cue, and the no-cue conditions, respectively. Response errors were larger in the termination-cue condition compared to the no-cue condition. Response errors decreased as the stimulus duration increased or the stimulus speed decreased. The error bars represent the standard error of means. Asterisks indicate statistical significance $(\mathrm{p}<0.05)$.

\section{Termination-cue effects depend on the stimulus duration and speed}

The above analyses showed that the termination-cue dilated the response duration, and the size of cue effects depended on the stimulus duration and speed. To explicitly visualize and analyze the nature of the interaction between the cue type and other factors, we directly examined cue effects, which were calculated by subtracting the response durations of the no-cue condition from those of the termination-cue or start-cue conditions. For the termination-cue condition, we merged the data from Experiments 1 and 2, considering that the termination-cue condition and the no-cue condition in Experiment 1 and Experiment 2 were identical. The possible confounding effect of the start-cue condition in Experiment 2 is 
expected to be minimal because the different conditions were performed on separate days.

A repeated measures ANOVA showed that the termination-cue effect (Fig. $4 \mathrm{a}$ and $4 \mathrm{~b}$, green line $)$ increased as the stimulus duration increased $\left(\mathrm{F}_{9,288}=11.02, \eta_{p}^{2}=.23, \mathrm{p}<.001\right)$ and decreased as the stimulus speed increased $\left(\mathrm{F}_{4,128}=18.73, \eta_{p}^{2}=.12, \mathrm{p}<.001\right)$. There was no interaction between the stimulus duration and speed $\left(\mathrm{F}_{36,1152}=1.08, \eta_{p}^{2}=.03, \mathrm{p}=.345\right)$, as depicted in Fig. 4c. We applied the same analyses to the start-cue condition and found qualitatively different results (Fig. $4 \mathrm{a}$ and $4 \mathrm{~b}$, red line). The duration effect was not significant $\left(\mathrm{F}_{9,153}=.94, \eta_{p}^{2}=.06, \mathrm{p}=.491\right)$. There was a significant speed effect $\left(\mathrm{F}_{4,68}=6.11\right.$, $\left.\eta_{p}^{2}=.09, \mathrm{p}<.001\right)$; however, the direction of the effect was opposite to the speed effect in the termination-cue condition. There was also a marginally significant interaction between duration and speed in Fig. $4 \mathrm{~d}\left(\mathrm{~F}_{36,612}=1.44, \eta_{p}^{2}=.08, \mathrm{p}=.049\right)$, which was not found in the termination-cue condition. Qualitatively different patterns between the termination-cue and start-cue effects demonstrated that the observed cue effects could not be due to the mere existence of a cue but involved the interaction between the cue and the moving clock hand. To formally describe cue effects, we considered three computational models in the next section.
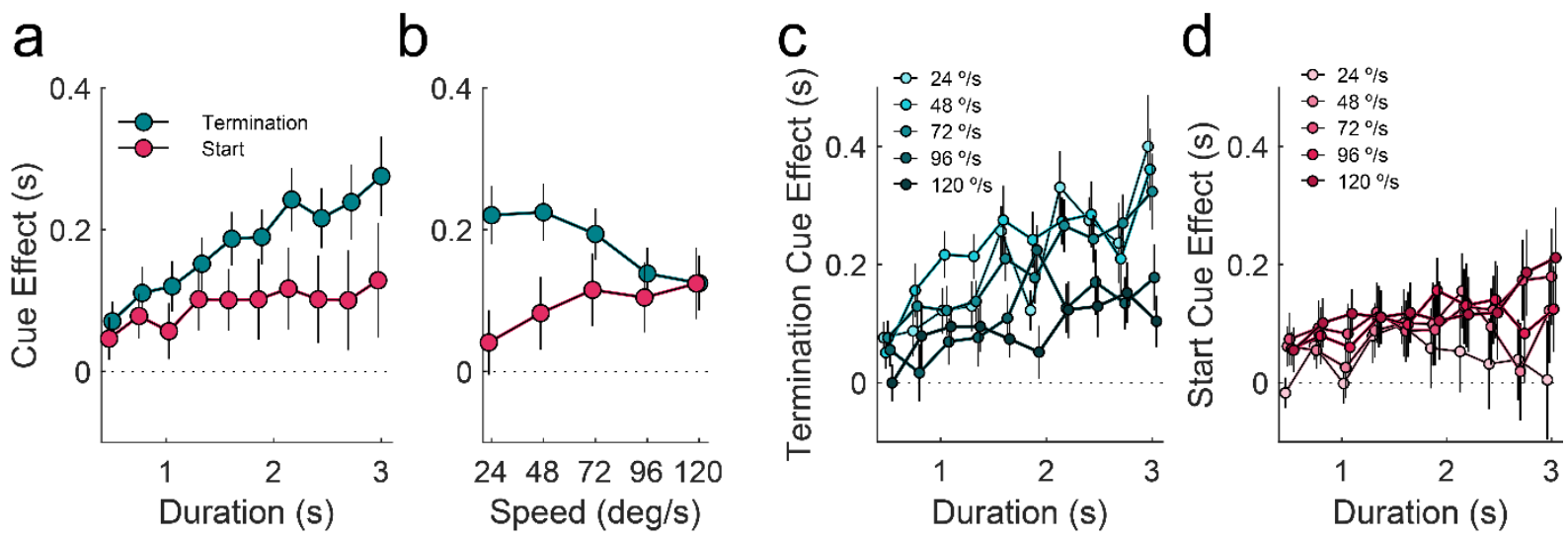

Fig. 4 Effects of the termination-cue and the start-cue. (a) Cue effects as a function of stimulus duration. Green and red colors indicate the termination-cue and the start-cue. (b) Cue effects as a function of the stimulus speed. (c) Termination-cue's effect as a function of 
the stimulus duration. The darker color matches with the faster speed whereas the brighter color matches with the slower speed. The effect of the termination-cue increased as the duration increased, or the speed decreased. (d) Start-cue's effect as a function of the stimulus duration. The effect of the start-cue increased as the speed increased. Note that the scales on ordinate of the left two panels and the right two panels are different. The error bars represent the standard error of means.

\section{Termination-cue generates a field where the perceived time dilates}

We developed models of cue effects inspired by the attention theory of time perception (Brown 1985; Wittmann, 2013). The theory claims that the subjective duration depends on the amount of attention allocated to the target object, relevant space, or task itself (Fortin et al., 1993; Mattes \& Ulrich, 1998). In our task, the termination-cue is likely to draw attention to the field near the cue, which may dilate the perceived duration. Because the field of attention can be formed in the domain of time or space (Coull \& Nobre, 1998; Coull \& Nobre, 2008; Rohenkohl et al 2014), the degree of attention, and consequently the degree of time dilation, can be a function of time or space, which we termed the time model and the space model, respectively. Additionally, we considered a third model in which the degree of time dilation is a function of space, but the time dilation is modulated by the time spent in the space. We termed the third model the time in space model (see Methods \& Supplementary Information). The time model predicts a functional relationship between stimulus duration and cue effect, and the space model predicts a functional relationship between stimulus distance and cue effect. In the time in space model, the cue effect is expressed as a function of the distance divided by the speed (Eq. 1). Thus, the model predicts a functional relationship between the stimulus distance and the cue effect multiplied by speed, which we called a speed-adjusted cue effect.

We plotted the observed cue effects or speed-adjusted cue effects against the stimulus duration or the stimulus distance (Fig. 5). How well the data points show a functional relationship indicates the model's goodness of fit. In the termination-cue condition (Fig. 5a- 
$5 c)$, the time in space model seems to manifest the functional relationship most clearly,

followed by the time model and the space model. The results of a formal model comparison supported the visual examination. We fitted a cubic function to cue effect functions $\left(F_{\text {Time }}\right.$, $F_{\text {Space }}$, and $\left.F_{\text {TinS }}\right)$ and computed the AIC of each model for individual participant's data. To compare the models' performances numerically, we computed the AIC differences between pairs of models for individuals and then bootstrapped 1000 samples of AIC differences. The time in space model was significantly better than the time model $\left(\mathrm{p}\left(\mathrm{AIC}_{\text {TinS }}<\mathrm{AIC}_{\text {Time }}\right)=\right.$ $0.992)$ and the space model $\left(\mathrm{p}\left(\mathrm{AIC}_{\mathrm{TinS}}<\mathrm{AIC}\right.\right.$ Space $\left.)>0.999\right)$. We applied the same analyses to the data from the start-cue condition. The time in space model provides a better fit than the time model $\left(\mathrm{p}\left(\mathrm{AIC}_{\text {TinS }}<\mathrm{AIC}_{\text {Time }}\right)=0.910\right)$ and the space model $\left(\mathrm{p}\left(\mathrm{AIC}_{\text {TinS }}<\mathrm{AIC}_{\text {Space }}\right)>\right.$ 0.999), although the difference between the time in space model and the time model was not statistically significant (see Supplementary Information for details).
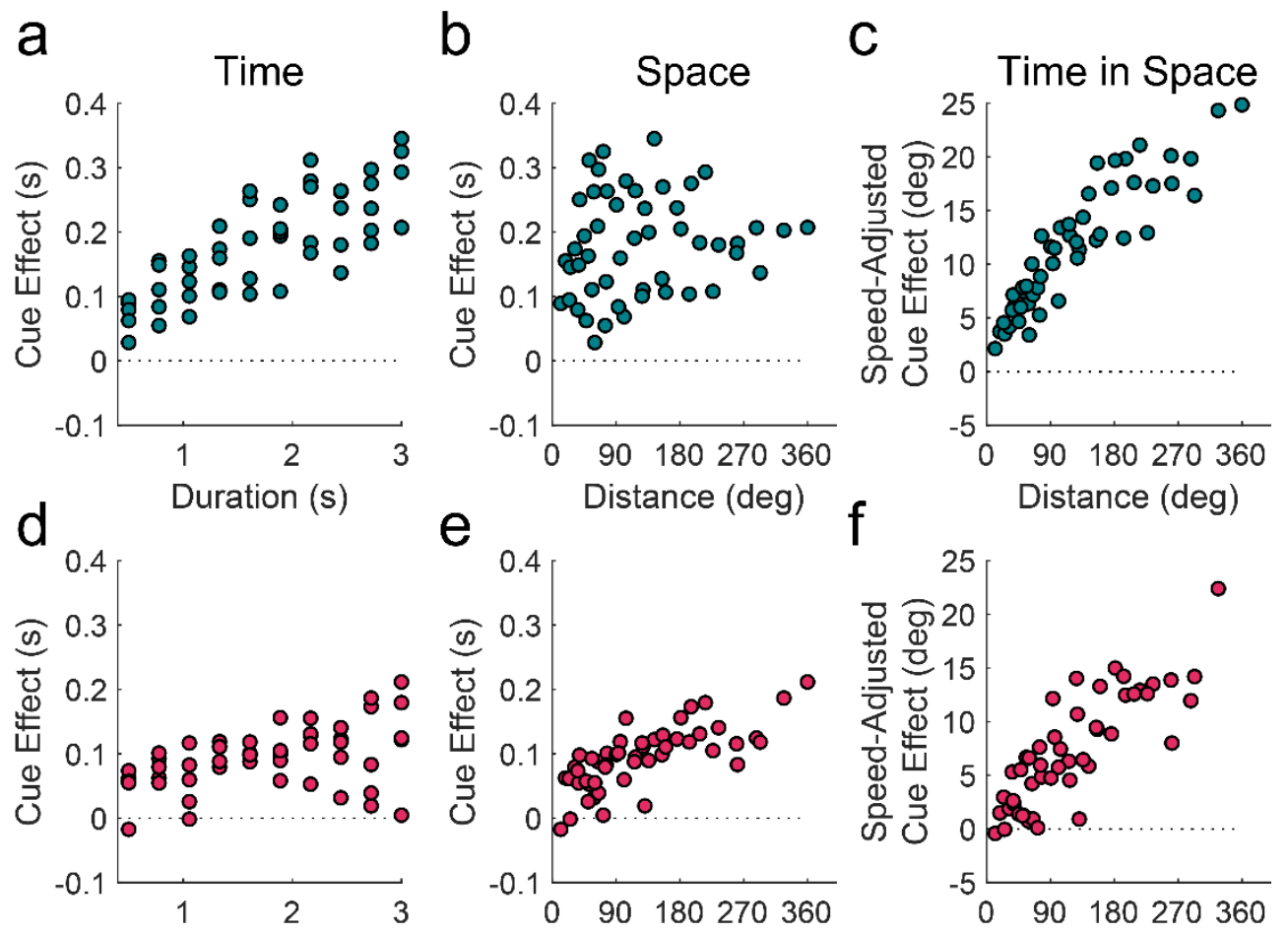

Fig. 5 Dependency between cue effects or speed-adjusted cue effect and the stimulus duration or distance: the time model (a and d), the space model (b and e), and the time in space (c and f) model. Note that abscissa and ordinate differ reflecting the prediction of each model. Green and red indicate the termination-cue condition and the start-cue condition, respectively. Each dot indicates the group mean over all participants' responses (termination- 
cue $=33$, start-cue $=18$ ) for a given distance and duration condition.

Figure 6 shows the performances of the time in space model. In the model, the cue effect function $F_{\text {Tins }}(d)$ indicates the cue effect when the travel distance is $d$ (Fig. 6a), and the derivative of it represents the accumulation rate of cue effects at each location, which is visualized in Fig. 6b. In the termination-cue condition, the accumulation rate was relatively high in the locations near the cue and decreased as the distance from the cue increased. To test the statistical significance of the pattern, we binned the distance into 10 levels and find that the accumulation rate was significantly different across distances $\left(\mathrm{F}_{9,288}=11.85, \eta_{p}^{2}\right.$ $=.27, \mathrm{p}<.001)$. The post-hoc test showed that bins of shorter distances had significantly higher dilation compared with bins of longer distances (Table S1), which suggests that the termination-cue generates a field of time dilation near the cue. In the start-cue condition, the accumulation rate did not show a significant change across distances $\left(\mathrm{F}_{9,153}=1.40, \eta_{p}^{2}=.08\right.$, $\mathrm{p}=.193$ ). In both conditions, the performance of the time in space model closely matched the empirical data displaying the systematic change of cue effects as a function of stimulus duration and speed (Fig. 6c and 6d). The other two models failed to capture the qualitative pattern of the cue effect (Fig. S1).
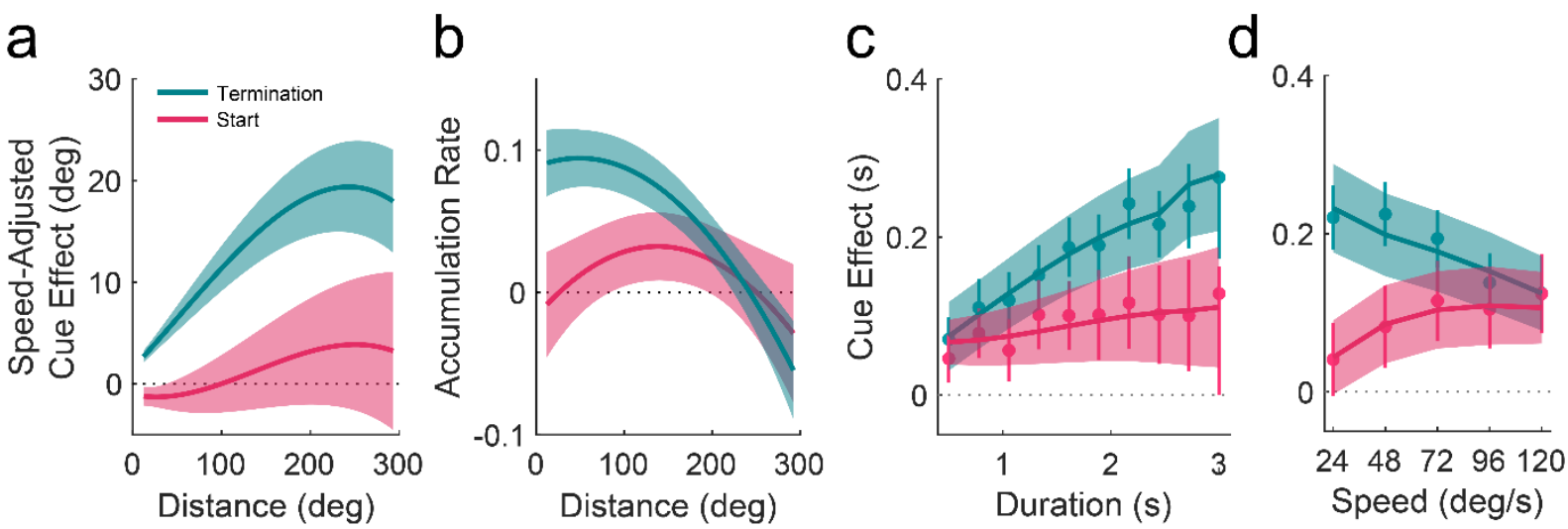

Fig. 6 Performances of the time in space model. (a) The reconstructed cue effect function $F_{\text {Tins }}(d)$ for termination-cue (green) and start-cue (red) conditions. (b) The accumulation rate as a function of the stimulus distance. In the termination-cue condition, the accumulation rate 
is highest near the cue and gradually decreases. In the start-cue condition, the accumulation rate remains almost steady. (c) The simulated cue effects across duration and (d) speed conditions closely matched the empirical data. The solid lines represent the estimated cue effects with the standard error of means (shaded area). The dots represent the empirical data with error bars (s.e.m.).

\section{Discussion}

Numerous studies have shown that the perceived duration of a time interval does not veridically reflect the physical duration but is distorted by various factors such as features of stimuli (Dormal et al., 2006; Oliveri et al., 2008), temporal context (Block \& Reed; 1978; Boltz, 1995), and the mental state of the observer (Angrilli et al., 1997; Katrin et al., 2015). Our results show that knowledge about an event's termination is another important factor. The perceived duration of a moving stimulus dilated when a cue indicating the termination point was presented, and the dilation effects increased as the stimulus duration increased, or the stimulus speed decreased. The observed pattern of termination-cue effects was qualitatively different from that of the start-cue effects, confirming that the information contained in the event's termination-cue indeed matters.

The attention theory of time perception can account for the observed results. The theory suggests that we perceive an event to last longer when attention is allocated to the event (Brown, 1985; Brown, 1997; Coull \& Nobre, 1998; Matthews \& Meck, 2016), and the time dilation caused by the attention allocation can be spatially localized (Mattes \& Ulrich, 1998). This theory suggests that the presence of the termination-cue, which is likely to attract attention, will increase the perceived duration of an event. This is what we observed in our study. In addition to empirically confirming the prediction of attention theory, the richness of our data allows us to further elaborate the theory. The existing attention theory of time perception is not specific enough to predict whether the size of the termination-cue effect is a function of time or space. Our results show an elaborated picture of time dilation caused by the termination-cue. The termination-cue generates a spatial field around the cue, and as an 
object moves in the spatial field, the perceived passage of time dilates. The size of dilation is determined by the distance from the termination-cue, and the dilation effect accumulates over time.

According to the Bayesian model of interval estimation, the ideal observer that optimizes the estimation performance exhibits a systematic bias toward the mean of the stimulus distribution (Agrillo et al., 2010; Jazayeri \& Shadlen, 2010; Miyazaki et al., 2005; Petzschner \& Glasauer, 2011). Our data are consistent with the model's prediction in that reproduced durations are biased toward the mean of distribution in no-cue conditions (Fig. 3ac). However, the pattern of cue effects is difficult to reconcile with the ideal observer model. The termination-cue is likely to assist the sensory measurements of the time interval and decrease the uncertainty of sensory inputs. Indeed, the termination-cue condition had reduced response variability compared with the no-cue condition (Experiment 1: $\mathrm{F}_{1,14}=$ $60.11, \eta_{p}^{2}=.48, \mathrm{p}<.001$; Experiment $\left.2: \mathrm{F}_{2,34}=6.54, \eta_{p}^{2}=.57, \mathrm{p}=.004\right)$. Because of the decreasing uncertainty of sensory inputs, the ideal observer model is expected to decrease the central tendency bias, contracting perceived durations in shorter duration stimuli, and dilating perceived durations in longer duration stimuli. Our results were not consistent with the prediction. The termination-cue dilated the perceived duration regardless of the stimulus duration.

We included the start-cue condition to test the possibility that the cue effect observed in the termination-cue condition was due to the physical existence of an additional stimulus, while the information regarding the event's termination is irrelevant. Our results clearly rule out this possibility by showing that the effects of the termination-cue and the start-cue differ not only in the magnitude but also in the pattern of the cue effects. However, the pattern of the start-cue effect itself, which increased as the stimulus speed increased, was unexpected. We conjecture that the start-cue might have assisted in the estimation of the travel distance 
that was positively correlated with the stimulus speed in our experiments. Accurate estimation of the distance will decrease the central tendency bias in distance estimation, which will effectively increase the perceived distance over a long distance and decrease it in a relatively short distance, modulating the perceived duration accordingly.

The explicit representation of the termination time by a location in space might have been responsible for the dilation field formed in space, and a different modality of the termination-cue may induce a dilation field in a domain other than space. The interaction between the modality of the termination-cue and the pattern of dilation remains to be explored. However, we conjecture that the main finding of the current study that the termination cue generates a field of time dilation may hold across modalities.

\section{Declarations}

\section{Acknowledgements}

This work was supported by the Ministry of Education of the Republic of Korea and the National Research Foundation of Korea (NRF-2020S1A3A2A02097375). The authors have no conflicts of interest to declare that are relevant to the content of this article.

\section{Author contributions}

S.C., and O.-S.K. designed research, performed research, analyzed data, and wrote the paper.

\section{Availability of data and materials}

Stimuli, data, and relevant codes for the experiments have been made publicly available via OSF and can be accessed at https://osf.io/ys7tg/. The design and analysis plans for the experiments were not preregistered. 


\section{References}

Agrillo, C., Ranpura, A., \& Butterworth, B. (2010). Time and numerosity estimation are independent: Behavioral evidence for two different systems using a conflict paradigm. Cognitive Neuroscience, 1(2), 96-101.

Allan, L. G. (1979). The perception of time. Perception \& Psychophysics, 26(5), 340-354.

Angrilli, A., Cherubini, P., Pavese, A., \& Manfredini, S. (1997). The influence of affective factors on time perception. Perception \& Psychophysics, 59(6), 972-982.

Assmus, A., Marshall, J. C., Noth, J., Zilles, K., \& Fink, G. R. (2005). Difficulty of perceptual spatiotemporal integration modulates the neural activity of left inferior parietal cortex. Neuroscience, 132(4), 923-927.

Bangert, A. S., Kurby, C. A., Hughes, A. S., \& Carrasco, O. (2020). Crossing event boundaries changes prospective perceptions of temporal length and proximity. Attention, Perception, \& Psychophysics, 82(3), 1459-1472.

Birngruber, T., Schröter, H., \& Ulrich, R. (2014). Duration perception of visual and auditory oddball stimuli: Does judgment task modulate the temporal oddball effect? Attention, Perception, \& Psychophysics, 76(3), 814-828.

Block, R. A., \& Reed, M. A. (1978). Remembered duration: Evidence for a contextualchange hypothesis. Journal of Experimental psychology: Human Learning and Memory, 4(6), 656.

Boltz, M. G. (1995). Effects of event structure on retrospective duration judgments. Perception \& Psychophysics, 57(7), 1080-1096.

Brainard, D. H., \& Vision, S. (1997). The Psychophysics Toolbox. Spatial Vision, 10(4), 433436.

Brown, S. W. (1985). Time perception and attention: The effects of prospective versus retrospective paradigms and task demands on perceived duration. Perception $\&$ 
Psychophysics, 38(2), 115-124.

Brown, S. W. (1995). Time, change, and motion: The effects of stimulus movement on temporal perception. Perception \& Psychophysics, 57, 105-116.

Brown, S. W. (1997). Attentional resources in timing: Interference effects in concurrent temporal and nontemporal working memory tasks. Perception \& Psychophysics, 59(7), $1118-1140$.

Buhusi, C. V., \& Meck, W. H. (2009). Relativity theory and time perception: Single or multiple clocks? PloS One, 4(7), e6268.

Cai, M. B., Eagleman, D. M., \& Ma, W. J. (2015). Perceived duration is reduced by repetition but not by high-level expectation. Journal of Vision, 15(13), 19-19.

Cai, Z. G., Wang, R., Shen, M., \& Speekenbrink, M. (2018). Cross-dimensional magnitude interactions arise from memory interference. Cognitive psychology, 106, 21-42.

Casasanto, D., \& Boroditsky, L. (2008). Time in the mind: Using space to think about time. Cognition, 106(2), 579-593.

Chang, C. J., \& Jazayeri, M. (2018). Integration of speed and time for estimating time to contact. Proceedings of the National Academy of Sciences, 115(12), E2879-E2887.

Cicchini, G. M., Arrighi, R., Cecchetti, L., Giusti, M., \& Burr, D. C. (2012). Optimal encoding of interval timing in expert percussionists. Journal of Neuroscience, 32(3), $1056-1060$.

Coull, J. T., \& Nobre, A. C. (1998). Where and when to pay attention: The neural systems for directing attention to spatial locations and to time intervals as revealed by both PET and fMRI. Journal of Neuroscience, 18(18), 7426-7435.

Coull, J. T., \& Nobre, A. C. (2008). Dissociating explicit timing from temporal expectation with fMRI. Current opinion in neurobiology, 18(2), 137-144.

Dormal, V., Seron, X., \& Pesenti, M. (2006). Numerosity-duration interference: A Stroop 
experiment. Acta Psychologica, 121(2), 109-124.

Droit-Volet, S., Brunot, S., \& Niedenthal, P. (2004). Perception of the duration of emotional events. Cognition and Emotion, 18(6), 849-858.

Faber, M., \& Gennari, S. P. (2015). In search of lost time: Reconstructing the unfolding of events from memory. Cognition, 143, 193-202.

Fortin, C., Rousseau, R., Bourque, P., \& Kirouac, E. (1993). Time estimation and concurrent nontemporal processing: Specific interference from short-term-memory demands. Perception \& Psychophysics, 53(5), 536-548.

Franssen, V., \& Vandierendonck, A. (2002). Time estimation: Does the reference memory mediate the effect of knowledge of results? Acta Psychologica, 109(3), 239-267.

Gibbon, J. (1977). Scalar expectancy theory and Weber's law in animal timing. Psychological Review, 84(3), 279.

Goldstone, S., \& Lhamon, W. T. (1974). Studies of auditory-visual differences in human time judgment: 1. Sounds are judged longer than lights. Perceptual and Motor Skills, 39(1), $63-82$.

Hollingworth, H. L. (1910). The central tendency of judgment. The Journal of Philosophy, Psychology and Scientific Methods, 7(17), 461-469.

Jazayeri, M., \& Shadlen, M. N. (2010). Temporal context calibrates interval timing. Nature Neuroscience, 13(8), 1020-1026.

Kaneko, S., \& Murakami, I. (2009). Perceived duration of visual motion increases with speed. Journal of Vision, 9(7), 14-14.

Kliegl, K. M., Watrin, L., \& Huckauf, A. (2015). Duration perception of emotional stimuli: Using evaluative conditioning to avoid sensory confounds. Cognition and Emotion, 29(8), 1350-1367.

Körding, K. P., \& Wolpert, D. M. (2004). Bayesian integration in sensorimotor 
learning. Nature, 427(6971), 244-247.

Kwon, O. S., \& Knill, D. C. (2013). The brain uses adaptive internal models of scene statistics for sensorimotor estimation and planning. Proceedings of the National Academy of Sciences, 110(11), E1064-E1073.

Lhamon, W. T., \& Goldstone, S. (1975). Movement and the judged duration of visual targets. Bulletin of the Psychonomic Society, 5(1), 53-54.

Li, Y., Mo, L., \& Chen, Q. (2015). Differential contribution of velocity and distance to time estimation during self-initiated time-to-collision judgment. Neuropsychologia, 73, 35-47.

Makin, A. D., Poliakoff, E., Dillon, J., Perrin, A., Mullet, T., \& Jones, L. A. (2012). The interaction between duration, velocity and repetitive auditory stimulation. Acta Psychologica, 139(3), 524-531.

Mattes, S., \& Ulrich, R. (1998). Directed attention prolongs the perceived duration of a brief stimulus. Perception \& Psychophysics, 60(8), 1305-1317.

Matthews, W. J., \& Meck, W. H. (2016). Temporal cognition: Connecting subjective time to perception, attention, and memory. Psychological bulletin, 142(8), 865.

Miyazaki, M., Nozaki, D., \& Nakajima, Y. (2005). Testing Bayesian models of human coincidence timing. Journal of Neurophysiology, 94(1), 395-399.

Oliveri, M., Vicario, C. M., Salerno, S., Koch, G., Turriziani, P., Mangano, R., Chillemi, G., \& Caltagirone, C. (2008). Perceiving numbers alters time perception. Neuroscience Letters, 438(3), 308-311.

Ono, F., \& Kawahara, J. I. (2007). The subjective size of visual stimuli affects the perceived duration of their presentation. Perception \& Psychophysics, 69(6), 952-957.

Pariyadath, V., \& Eagleman, D. (2007). The effect of predictability on subjective duration. PloS One, 2(11), e1264.

Pelli, D. G., \& Vision, S. (1997). The VideoToolbox software for visual psychophysics: 
Transforming numbers into movies. Spatial Vision, 10, 437-442.

Petzschner, F. H., \& Glasauer, S. (2011). Iterative Bayesian estimation as an explanation for range and regression effects: A study on human path integration. Journal of Neuroscience, 31(47), 17220-17229.

Rohenkohl, G., Gould, I. C., Pessoa, J., \& Nobre, A. C. (2014). Combining spatial and temporal expectations to improve visual perception. Journal of vision, 14(4), 8-8.

Schirmer, A. (2011). How emotions change time. Frontiers in Integrative Neuroscience, 5 , 58.

Stetson, C., Fiesta, M. P., \& Eagleman, D. M. (2007). Does time really slow down during a frightening event? PloS One, 2(12), e1295.

Tipples, J. (2008). Negative emotionality influences the effects of emotion on time perception. Emotion, 8(1), 127.

Tse, P. U., Intriligator, J., Rivest, J., \& Cavanagh, P. (2004). Attention and the subjective expansion of time. Perception \& Psychophysics, 66(7), 1171-1189.

Ulrich, R., Nitschke, J., \& Rammsayer, T. (2006). Perceived duration of expected and unexpected stimuli. Psychological Research, 70(2), 77-87.

Wearden, J. H., Todd, N. P. M., \& Jones, L. A. (2006). When do auditory/visual differences in duration judgements occur? Quarterly Journal of Experimental Psychology, 59(10), 1709-1724.

Witherspoon, D., \& Allan, L. G. (1985). The effect of a prior presentation on temporal judgments in a perceptual identification task. Memory \& Cognition, 13(2), 101-111.

Wittmann, M. (2013). The inner sense of time: How the brain creates a representation of duration. Nature Reviews Neuroscience, 14(3), 217-223.

Xuan, B., Zhang, D., He, S., \& Chen, X. (2007). Larger stimuli are judged to last longer. Journal of Vision, 7(10), 2-2. 